\title{
Reliability of a New Test of Levator Scapula Muscle Length
}

\author{
Young-mun Song ${ }^{1}$; Jong-hyuck Weon, PT, Ph.D²; Do-young Jung, PT, Ph.D ${ }^{2}$ \\ ${ }^{1}$ Jeonju Drim Sol Hospital, Jeonju, South Korea \\ ${ }^{2}$ Department of Physical Therapy, College of Health \& Welfare, Joongbu University, Geumsan, South Korea
}

Background Shortened levator scapula (LS) is a major cause of neck and shoulder pain and reduced motor function. Therefore, measuring LS muscle length is a key element in evaluating patients with shoulder dysfunction.

Purpose The purpose of this study was to evaluate the intra- and inter-rater reliability of a newly designed levator scapular length test (LSLT).

Study design Intra- and inter-tester repeated measures.

Methods We recruited 28 participants in this study. Subjects were divided into two groups: normal scapula length and shortened scapula length. The LS index (LSI) and LSLT were applied twice by one examiner and once by a different examiner. The intraclass correlation coefficient was used to assess intra- and inter-rater reliability of the LSI and LSLT. Pearson's correlation coefficient was used to evaluate the correlation between the tests.

Results The intra-rater reliability scores of LSI and LSLT were 0.95 and 0.98 , and the inter-rater test reliability scores were 0.96 and 0.98 , respectively. The correlation coefficient was 0.63 , indicating a moderate relationship between tests.

Conclusions These findings suggest that LSLT is a reliable method for measuring LS length in clinical practice.

Key words Levator scapular index; Levator scapular length test; Muscle length; Reliability.

JMST

2019; 3(1): 1-6

Published Online Jun 30, 2019 pISSN 2635-8573 eISSN 2635-8581

Article History

Received 03 April 2019

Revised 04 April 2019

(1st)

Revised 18 April 2019

(2nd)

Accepted 27 April 2019

\section{CONTACT}

ptsports@joongbu.ac.kr

Do-young Jung,

Department of Physical

Therapy, College of

Health \& Welfare,

Joongbu University,

Geumsan, South Korea

This is an Open-Access article distributed under the terms of distributed under the terms of the Creative Commons Attribution Non-Commercial License (http://creativecommons org/licenses/by-nc/4.0) which permits unrestricted non-commercial use, distribution, and reproduction in any medium, provided the original work is properly cited.

\section{INTRODUCTION}

The levator scapula (LS) originates at the transverse processes of four upper cervical vertebrae and inserts diagonally into the superior angle of the scapula. The LS elevates and rotates the scapula downward. ${ }^{1-3}$ As the LS becomes shorter, the superior angle of the scapula rises above normal alignment, giving the entire girdle an elevated appearance. ${ }^{2}$ Shortened LS contributes to stiffness during flexion and contralateral rotation of the neck. ${ }^{4}$ Dominance and shortness of the LS are accompanied by weakness and lengthening of its antagonists such as the upper trapezius (UT), lower trapezius (LT), and serratus anterior (SA). ${ }^{2,5-6}$
Increased stiffness or shortness of the LS can contribute to increased compressive load and shear force on the cervical spine during active neck movement and shoulder abduction, leading to pain or impairment of motor function. ${ }^{7-9}$

Sahrmann S. ${ }^{2}$ divided impairments of abnormal scapular alignment into several categories. Among these, downward scapular rotation is the most common impairment in patients with shoulder pain; it is characterized by shortening of the LS and rhomboid and lengthening of the UT and SA. In scapular elevation syndromes, LS shortening acts as a major factor in abnormal movement of the scapula. To raise the arm to $180^{\circ}$ with slight depression and posterior tilt, adduction of the scapula should occur in the end range. 
However, when the LS is shortened, the scapula is elevated rather than depressed in the end range, and cervical rotation and lateral displacement are increased because the flexibility of the cervical spine exceeds the extensibility of the LS. ${ }^{2}$ Fatigue or weakness of the LT and SA accompanied by LS shortening can cause shoulder impingement due to insufficient upward scapular rotation. ${ }^{10-12}$ Therefore, measuring the LS muscle length should be a key element in evaluating shoulder dysfunction. Previous studies have proposed several methods for evaluating LS muscle length. Behrsin and Maguire $^{7}$ measured LS muscle length as the distance from the transverse process of the first cervical vertebra to the superior angle of the scapula using an X-ray. Lee et al. ${ }^{13}$ measured LS muscle length directly using calipers. Janda V. ${ }^{14}$ proposed evaluating the degree of LS resistance experienced during cervical passive movement as a measure of LS length. However, previous studies have had several limitations. X-ray measurements of LS were affected by distortion in the two-dimensional images. ${ }^{7}$ Caliper measurements require skill to accurately palpate the anatomical position of the LS origin and insertion points. ${ }^{13}$ The degree of LS resistance is difficult to evaluate objectively using the method suggested by Janda. ${ }^{14}$ Therefore, it is necessary to develop a clinically simple and reliable LS measurement method. The purpose of this study was to determine the reliability of a new method of measuring LS muscle length.

\section{METHODS}

\section{Participants}

We recruited 28 adults to participate in this study. Both shoulders were used to measure LS muscle length. All participants voluntarily participated and signed a consent form prior to the study, after listening to an explanation of the purpose and method of the experiment. Subject inclusion criteria were as follows: 1) no limitation of active shoulder movement; 2) no instability such as subluxation of the humeral head while lifting the arm; 3) no history of shoulder surgery; and 4) no shoulder or neck pain during active shoulder flexion.

The protocol of this study was approved by the Institutional Review Board (IRB) of Joongbu University (JIRB2017051901-02-170612). Subjects were divided into two groups (normal LS and shortened LS) following an active shoulder flexion test (ASFT). Subjects were assigned to the shortened group if the neck was rotated or scapular superior angle medial border elevated in the end range during shoulder flexion. Subjects were assigned to the normal group if the scapular inferior angle moved at the midaxillary line with little depression and posterior adduction of the scapula at the end range during shoulder flexion. Following the screening test, 11 subjects were selected for the normal group, and 17 subjects for the shortened LS group. One shoulder was excluded from the normal group due to shoulder instability. Four shoulders were excluded from the shortened LS group due to shoulder pain and shoulder instability. Therefore, this study included 21 shoulders in the normal group and 30 shoulders in the shortened LS group. Descriptive characteristics of the subjects are presented in Table 1.

\section{Procedure}

Three measurements were performed to measure LS length, including the ASFT, LS index (LSI) using calipers, and our newly developed LS length test (LSLT). Experiments were performed by two examiners, each of whom had more than 4 years of clinical experience in a private physical therapy clinic, and one assistant who took photographs of the experiment. The examiners participated in a 3hour training study to become familiar with the precise locations of the bony landmarks used in this study and to reduce measurement error. The LSI and LSLT were performed twice by one examiner and once by the other examiner. The ASFT was applied once by a single examiner. A rest period was provided between tests. Measurements were determined by the examiner and from photographs. After all tests were complete, photograph angles were calculated using software. The orders of measurements and examiners were randomized by drawing lots, and both examiners were blinded to the measurement results (Figure 1).

\section{1) ASFT measurements}

The ASFT was performed to measure shoulder flexion ROM. First, two markers (diameter, $5 \mathrm{~mm}$ ) were attached to the lateral epicondyle of the humerus and the central axis of shoulder rotation in a sitting position. Subjects were then asked to perform the ASFT with no trunk extension and no neck or scapular fixation. Photographs were taken at a distance of $1 \mathrm{~m}$ from the subject, with the tripod height

Table 1. General characteristics of subjects

$(\mathrm{N}=28)$

\begin{tabular}{ccc}
\hline Variable & $\begin{array}{c}\text { Normal group } \\
(\mathrm{n}=11)\end{array}$ & $\begin{array}{c}\text { Shortened group } \\
(\mathrm{n}=17)\end{array}$ \\
\hline Age $(\mathrm{yrs})$ & $22.86 \pm 2.24^{\mathrm{a}}$ & $21.27 \pm 1.64$ \\
Height $(\mathrm{cm})$ & $161.81 \pm 6.62$ & $163.87 \pm 6.66$ \\
Weight $(\mathrm{kg})$ & $57.81 \pm 13.27$ & $57.57 \pm 8.63$ \\
\hline
\end{tabular}

${ }^{a}$ Mean \pm standard deviation. 


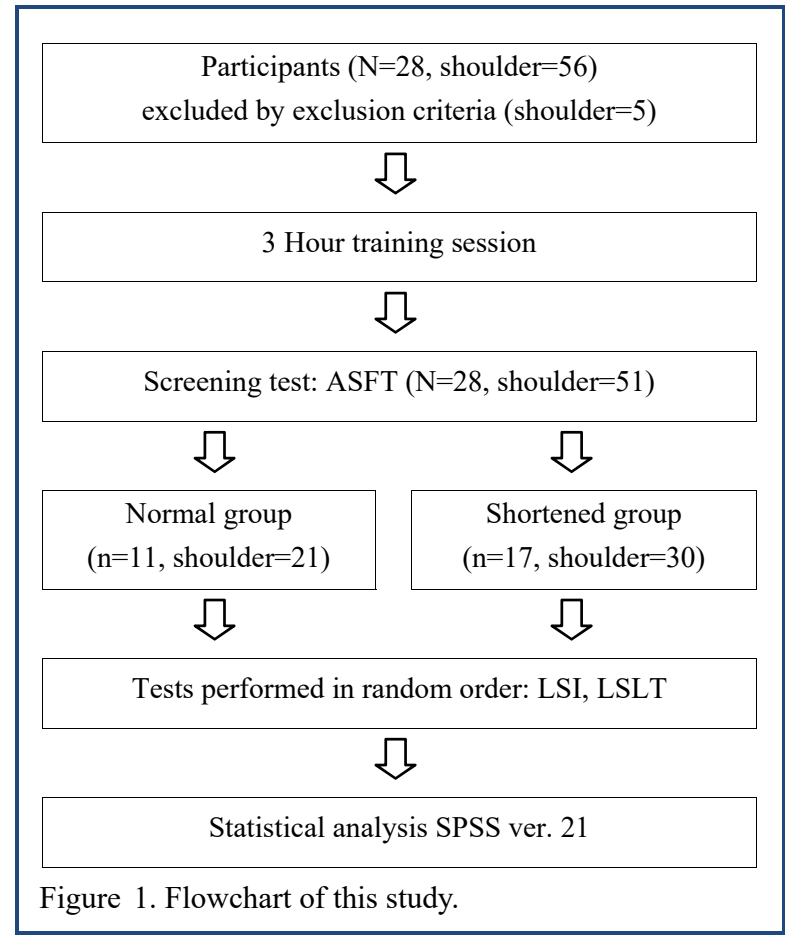

horizontally aligned with subject's shoulder. Shoulder flexion ROM was measured using photogrammetry postural analysis.

\section{2) LSI measurements}

Because the LS muscle lies between the cervical spine and scapula, it is influenced by neck position. Therefore, the examiner instructed the subject to maintain a neutral position in the craniocervical spine in the frontal, sagittal, and horizontal planes before measurement. Subjects were asked to hold their breath during measurements to limit the effect of breathing on LS length. To measure LS muscle length, the examiner palpated two scapular bony prominences: the dorsal tubercles of the transverse process of the second cervical vertebra and the superior angle of the medial border of the scapula; ${ }^{7}$ markers (diameter $0.5 \mathrm{~cm}$ ) were attached at these two bony prominences. The examiner measured the distance between these two landmarks using digital calipers (Absolute Digimatic Caliper, Mitutoyo, Japan). Following these measurements, the markers were immediately removed so that they would not be visible in subsequent tests. Measurements were converted into LSI as LS length $(\mathrm{cm}) /$ subject height $(\mathrm{cm}) \times 100$.

\section{3) LSLT measurements}

Prior to LSLT measurements, two markers (diameter 5 $\mathrm{mm}$ ) were attached to the lateral epicondyle of the humerus and the central axis of shoulder rotation with the subject in a sitting position supported by a vertical pillar. ${ }^{15,16}$ At the time of measurement, the examiner flexed the head to induce lateral bending and rotation in the contralateral direction until end feel of the elongated LS. The examiner then lightly pressed the medial part of the scapula with the other hand to prevent the superior angle of the scapula from rising during the test (Figure 2B). ${ }^{4,14}$ In this posture, the subject was instructed to flex the shoulder joint until resistance and muscle tightness were felt. Photographs were taken at a distance of $1 \mathrm{~m}$ from the subject, with the tripod height horizontally aligned with subject's shoulder. Shoulder flexion ROM was calculated using NIH Image J software (National Institute of Health, Bethesda, Maryland, USA) as the angle between a vertical line along the central axis of shoulder rotation and the lateral epicondyle of the humerus (Figure 2A).

\section{Data analysis}

LSI and LSLT intra- and inter-rater reliability were calculated using the intraclass correlation coefficient $\left(\mathrm{ICC}_{3,1}\right)$.
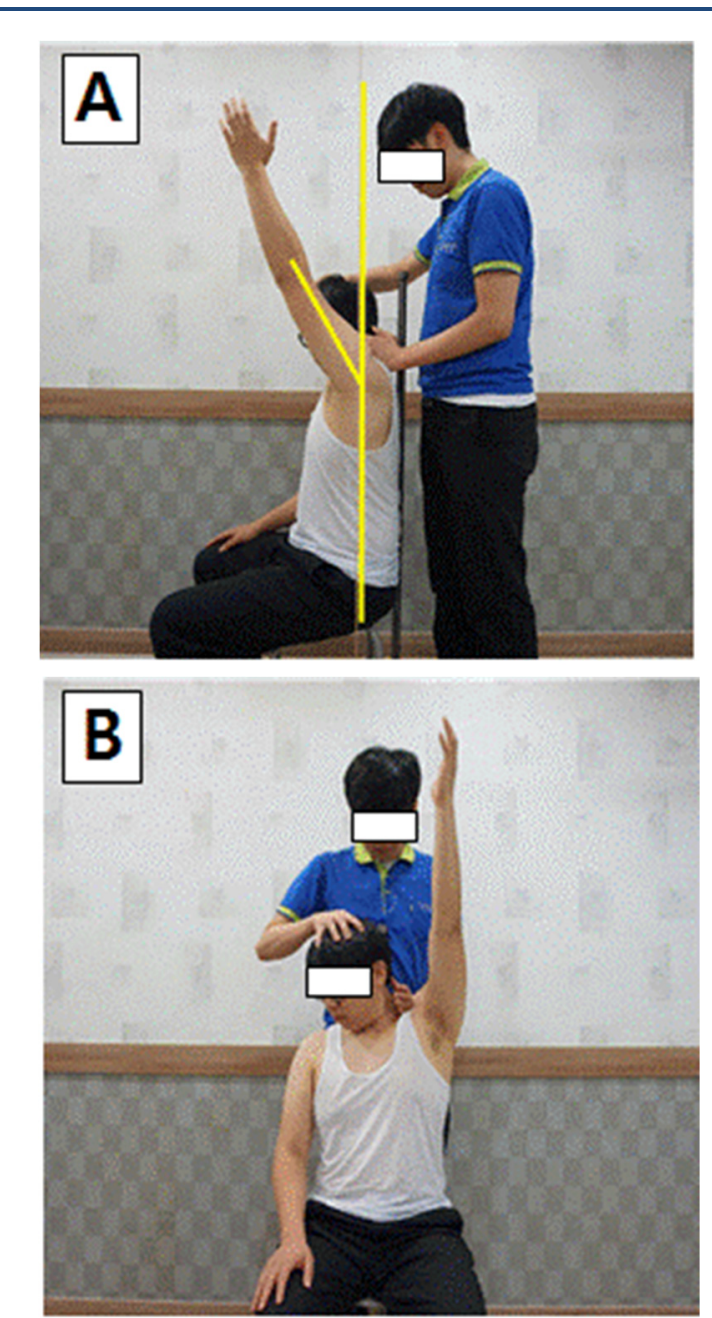

Figure 2. Levator scapulae length test (A: side view, B: frontal view). 
Pearson's correlation coefficient was used to evaluate the correlation between LSI and LSLT results. An independentsamples Student's t-test was used to determine differences in shoulder joint flexion angle during ASFT and LSLT between groups. A paired $t$-test was used to determine differences in ASFT and LSLT results between groups. SPSS v. 21 software was used for statistical analyses, with the level of statistical significance set at $p<0.05$.

\section{RESULTS}

The intra-rater reliability of the LSI and LSLT were 0.95 and 0.98 , respectively, and inter-rater test reliability was 0.96 and 0.98 , respectively. There was significant relationship between LSI and LSLT $(r=0.63, p<0.05)$. The LSLT results showed that the shortened LS group showed significantly lower shoulder flexion ROM than the normal group $(p<0.05)$. No significant difference in shoulder flexion ROM during the ASFT was detected between groups $(p<0.05)$ (Table 2). No significant difference in shoulder flexion ROM between the ASFT and LSLT was detected in the normal group $(p>0.05)$. However, in the shortened LS group, shoulder flexion ROM was significantly lower during LSLT than during ASFT $(p<0.05)$ (Table 2).

\section{DISCUSSION}

The intra-rater reliability scores of the LSI and LSLT were 0.96 and 0.98 , respectively, and the inter-rater reliability scores of the LSI and LSLT were 0.95 and 0.97 , respectively. Lee et al. ${ }^{13}$ also reported high intra-rater and inter-rater reliability for the LSI (0.94 and 0.82, respectively). These results indicate that both LSI and LSLT have the high reliability required for LS length evaluation. The

Table 2. Comparison of range of motion measured by two measurement methods between normal and shortened groups

$(\mathrm{n}=51$ shoulders $)$

\begin{tabular}{cccc}
\hline & Normal group & Shortened group & $p$-value \\
\hline ASFT & $172.48 \pm 2.68$ & $171.86 \pm 2.69$ & .43 \\
LSLT & $172.28 \pm 3.66$ & $148.58 \pm 7.25$ & $.00^{*}$ \\
$p$-value & .72 & .00 & \\
\hline
\end{tabular}

${ }^{*}$ Significance of difference of shoulder flexion ROM between groups in each test.

${ }^{\ddagger}$ Significance of difference of shoulder flexion ROM between tests within group.

Abbreviation: ASFT, active shoulder flexion test; LSLT, levator scapulae length test. correlation coefficient was 0.63 , showing a moderate relationship between the LSLT and LSI.

There was no significant difference in shoulder flexion ROM during ASFT between groups (control, 172.48 ; shortened LS, $171.86^{\circ} ; p>0.05$ ). Bruce $\mathrm{R} .{ }^{17}$ reported that the normal range for shoulder flexion should exceed $160^{\circ}$, consistent with the results of this study. However, the LSLT showed a significant difference in shoulder flexion ROM between the control group $\left(172.28^{\circ}\right)$ and the shortened LS group $\left(148.58^{\circ}\right)$; therefore, it is difficult to determine whether the LS is shortened using the ASFT. We also found a significant difference in shoulder flexion ROM between the ASFT and LSLT in the shortened LS group, but not in the normal group. Based on these results, we conclude that the LSLT is an appropriate evaluation tool for assessing LS length.

The LSLT was designed to induce passive insufficiency by holding the neck and scapula in position while the arm is raised, thereby stretching the LS as much as possible. This results in an inability to lift the arm completely due to the tension created by the shortened LS. Kendall et al. ${ }^{1}$ proposed that to accurately measure muscle length, movements should be performed with maximum muscle elongation. Thus, to determine the maximum extent to which the muscle can be stretched, the ROM of the joint should be measured by performing passive or active movement to increase the distance between the origin and the insertion. The origin of the muscle is thus fixed during the stretching movement, and its insertion is moved in the direction opposite to the muscle action. Therefore, in this study, we measured LS length by flexing the head in the opposite direction by applying lateral bending and rotation until end feel. To upwardly rotate the scapula in the direction of LS muscle elongation, active shoulder joint flexion was performed. While the shoulder joint was flexed, shoulder flexion ROM was measured in a state that prevented excessive adduction of the shoulder or elevation of the superior angle of the scapula.

During the LSLT, the examiner pressed lightly on the superior angle of the scapula to prevent elevation of the scapula during arm elevation. Bagg and Forrest ${ }^{18}$ reported rotation of the scapula starting from the scapular spine root to the acromioclavicular joint during upward scapular rotation. In early shoulder abduction, the acromion is elevated, and the medial border of the scapula is depressed at the end range of shoulder abduction. To flex the shoulder to $180^{\circ}$, the scapula should be upwardly rotated by about $60^{\circ}$; this movement causes elongation of the LS and downward rotation of the scapula. However, it has been reported that the LS does not extend sufficiently if the 
superior angle of the scapula is elevated or the neck is rotated at the end range of shoulder flexion. ${ }^{2,19}$ The LSLT was performed while preventing compensatory action such as increased superior angle of the scapula or rotation of the neck, which was observed at the end range of shoulder flexion in the shortened LS group. This result may explain why shoulder flexion ROM during the LSLT was lower in the shortened LS group than in the control group.

This study has some limitations. First, because the participants were healthy adults, it may be difficult to generalize the results of this study. Second, we did not validate our LSLT results. Shoulder flexion angle ROM was measured using a technique that was convenient for clinical practice to address the difficulty of measuring upward scapular rotation. In subjects with glenohumeral joint instability, LSLT sensitivity may be low. Future studies should apply a direct method to measure upward rotation of the scapula to determine whether the LS is shortened.

\section{CONCLUSION}

The purpose of this study was to evaluate the intra- and inter-rater reliability of LSI and a newly designed LSLT for measuring LS length. Our results show that both LSLT and LSI have the high reliability required for LS length evaluation. The correlation coefficient between the two was 0.63 , indicating a moderate relationship. Therefore, we recommend the LSLT as a simple technique to evaluate LS muscle length in clinical practice.

\section{Key Points}

Question What is the reliability and validity of a newly designed levator scapular length test (LSLT)?

Findings The intra- and inter-rater reliability of levator scapular index (LSI) and range of active shoulder flexion by taken by LSLT were excellent.

The range of active shoulder flexion by taken by LSLT was moderate correlated with LSI.

Meaning The LSLT is a highly reliable and valid tool for levator scapular length measurement.

\section{Article information}

Conflict of Interest Disclosures: None.

Funding/Support: None.

Acknowledgment: None.

Ethic Approval: The study received the approval of the Institute Review Board (IRB) of Joongbu University.

\section{REFERENCES}

1. Kendall FP, McCreary EK, Provance PG, et al. Muscles: testing and function with posture and pain. 5th ed. Baltimore: Williams \&Wilkins; 2005;1-358.

2. Sahrmann S. Diagnosis and treatment of movement impairment syndromes. Elsevier Health Sciences. Mosby; 2001.

3. Mottram SL. Dynamic stability of the scapula. Man Ther. 1997;2(3):123-131.

4. Diener I. The effect of levator scapula tightness on the cervical spine: Proposal of another length test. Journal of Manual \& Manipulative Therapy. 1998;6(2):78-86.

5. Grant R. Physical therapy of the cervical and thoracic spine. Churchill Livingstone; 1994;1-442.

6. Neumann DA. Kinesiology of the musculoskeletal system: foundations for rehabilitation. St Louis, MO: Mosby. Elsevier; 2010;119-172.

7. Behrsin JF, Maguire K. Levator scapulae action during shoulder movement: a possible mechanism for shoulder pain of cervical origin. Aust J Physiother. 1986;32(2): 101-106.

8. Szeto GP, Straker L, Raine S. A field comparison of neck and shoulder postures in symptomatic and asymptomatic office workers. Appl Ergon. 2002;33(1):75-84.

9. Van Dillen LR, McDonnell MK, Susco TM, Sahrmann SA. The immediate effect of passive scapular elevation on symptoms with active neck rotation in patients with neck pain. Clin J Pain. 2007;23(8):641-647.

10. Kibler BW, McMullen J. Scapular dyskinesis and its relation to shoulder pain. J Am Acad Orthop Surg. 2003;11(2):142-151.

11. McQuade KJ, Dawson J, Smidt GL. Scapulothoracic muscle fatigue associated with alterations in scapulohumeral rhythm kinematics during maximum resistive shoulder elevation. J Orthop Sports Phys Ther. 1998; 28(2):74-80.

12. Phadke V, Ludewig PM. Study of the scapular muscle latency and deactivation time in people with and without shoulder impingement. $J$ Electromyogr Kinesiol. 2013;23(2):469-475.

13. Lee JH, Cynn HS, Choi WJ, Jeong HJ, Yoon TL. Reliability of levator scapulae index in subjects with and without scapular downward rotation syndrome. Phys Ther Sport. 2016;19:1-6.

14. Janda V. Muscle and cervicogenic pain syndromes. Physical therapy for the cervical and thoracic spine. Churchill Livingstone; 1988;153-166.

15. Norkin JW, White J, Ostrow AC. Measurement of joint motion: a guide to goniometry. Davis FA; 1995;66-114. 
16. Yang JL, Lu TW, Chou FC, Chang CW, Lin JJ. Secondary motions of the shoulder during arm elevation in patients with shoulder tightness. $J$ Electromyogr Kinesiol. 2009;19(6):1035-1042.

17. Bruce R. The orthopaedic physical examination. Elsevier Saunders; 2005;17-66.
18. Bagg SD, Forrest WJ. A biomechanical analysis of scapular rotation during arm abduction in the scapular plane. Am J Phys Med Rehabil. 1988;67(6):238-245.

19. Ludewig PM, Cook TM, Nawoczenski DA. Threedimensional scapular orientation and muscle activity at selected positions of humeral elevation. J Orthop Sports Phys Ther. 1996;24(2):57-65. 